Pesq. Vet. Bras. 30(1):13-20, janeiro 2010

\title{
Intoxicações por plantas diagnosticadas em ruminantes e equinos e estimativa das perdas econômicas na Paraíba ${ }^{1}$
}

\author{
Tales S. Assis², Rosane M.T. Medeiros²*, Franklin Riet-Correa², Glauco J.N. \\ Galiza$^{2}$, Antônio F.M. Dantas ${ }^{2}$ e Diego M. Oliveira²
}

\begin{abstract}
Assis T.S., Medeiros R.M.T., Riet-Correa F., Galiza G.J.N., Dantas A.F.M. \& Oliveira M.D. 2010. [Plant poisonings diagnosed in ruminants and horses and estimation of the economical losses in Paraíba.] Intoxicações por plantas diagnosticadas em ruminantes e equinos e estimativa das perdas econômicas na Paraíba. Pesquisa Veterinária Brasileira 30(1):13-20. Hospital Veterinário, Centro de Saúde e Tecnologia Rural, Campus de Patos, Universidade Federal de Campina Grande, Patos, PB 58700-000, Brazil. E-mail: rmtmed@uol.com.br

This paper reports plant poisonings in ruminants and horses, diagnosed between 2000 and 2007, in the Veterinary Pathology Laboratory at the Federal University of Campina Grande, in the city of Patos, state of Paraíba. In cattle, $7.4 \%$ of the diseases diagnosed were caused by poisonous plants. Outbreaks were caused by Centhraterum brachylepis (1), Brachiaria spp. (1), Crotalaria retusa (2), Ipomoea batatas (1), Marsdenia sp. (1), grass containing nitrites (3 outbreaks, 1 by Echinochloa polystachya and 2 by Pennisetum purpureum), Palicourea aeneofusca (1), Prosopis juliflora (3), Nerium oleander (1), and Mimosa tenuiflora (7). In sheep, $13 \%$ of the diseases diagnosed were caused by toxic plants. Four outbreaks were caused by Ipomoea asarifolia, 3 by Brachiaria spp., 2 by Crotalaria retusa, 2 by Tephrosia cinerea, 1 by Panicum dichotomiflorum, 1 by Mascagnia rigida, and 20 by Mimosa tenuiflora. In goats, $6.4 \%$ of the diseases were caused by toxic plants. Seven outbreaks were caused by Mimosa tenuiflora, 1 by Ipomoea asarifolia, 1 by Ipomoea carnea, 1 by Ipomoea riedelli, 3 by Prosopis juliflora, 1 by Arrabidaea corallina, 2 by Aspidosperma pyrifolium, and 2 by Turbina cordata. In horses, $14 \%$ of the diagnosed diseases were due to plants poisonings including 12 outbreaks caused by Crotalaria retusa and one by Turbina cordata. Annual losses in the state of Paraíba by deaths of livestock are estimated in 3,895 cattle, 8,374 sheep, 6,390 goats, and 366 horses, which represent about US\$1,380,000. Epidemiologic, clinical and pathologic aspects of poisonings by Crotalaria retusa in cattle, Brachiaria spp. in sheep, Prosopis juliflora in cattle and goats, Nerium oleander in cattle, Opuntia ficus-indica in goats, and Turbina cordata in horses and goats are reported.
\end{abstract}

INDEX TERMS: Poisonous plants, plant poisoning, cattle, sheep, horses, goats, economic losses, semiarid.

RESUMO.- Foi realizado um levantamento dos surtos de intoxicações por plantas em ruminantes e equinos diagnosticados no Laboratório de Patologia Veterinária (LPV), do Hospital Veterinário da Universidade Federal de Campina Grande, Campus de Patos, Paraíba, no período de

\footnotetext{
${ }^{1}$ Recebido em 22 de abril de 2009.

Aceito para publicação em 15 de agosto de 2009

2 Hospital Veterinário, Centro de Saúde e Tecnologia Rural, Universidade Federal de Campina Grande, Campus de Patos, Patos, PB 58700970, Brasil. *Autor para correspondência:rrmtmed@uol.com.br
}

2000-2007. Em bovinos 7,4\% dos diagnósticos realizados pelo LPV foram intoxicações por plantas. Foram diagnosticadas intoxicações por Centhraterum brachylepis (um surto), Brachiaria spp. (um surto), Crotalaria retusa (dois surtos), Ipomoea batatas (um surto), Marsdenia sp. (um surto), gramíneas contendo nitratos e nitritos (um surto por Echinochloa polystachya e dois surtos por Pennisetum purpureum), Palicourea aeneofusca (um surto), Prosopis juliflora (três surtos), Nerium oleander (um surto) e Mimosa tenuiflora (sete surtos). Na espécie ovina 13\% dos diagnósticos foram intoxicações por plantas. Os surtos foram 
causados por Ipomoea asarifolia (quatro surtos), Brachiaria spp. (três surtos), Crotalaria retusa (dois surtos), Tephrosia cinerea (dois surtos), Panicum dichotomiflorum (um surto), Mascagnia rigida (um surto) e malformações associadas à ingestão de Mimosa tenuiflora (20 surtos). Nos caprinos, $6,4 \%$ dos diagnósticos corresponderam à intoxicação por plantas. Sete surtos foram causados por Mimosa tenuiflora, um por Ipomoea asarifolia, um por Ipomoea carnea, um por Ipomoea riedelli, três por Prosopis juliflora, um por Arrabidaea corallina, dois por Aspidosperma pyrifolium, dois por Turbina cordata e um por Opuntia ficus-indica. Na espécie equina $14 \%$ das doenças diagnosticadas foram devidas a intoxicações por plantas, sendo 12 surtos por Crotalaria retusa e um por Turbina cordata. As perdas na Paraíba por plantas tóxicas são estimadas em 3.895 bovinos, 8.374 ovinos, 6.390 caprinos e 366 equinos, que representam uma perda econômica anual, por morte de animais, de $\mathrm{R} \$ 2.733 .097,00$. São relatados alguns aspectos epidemiológicos, sinais clínicos e patologia de surtos de intoxicação por Crotalaria retusa em bovinos, Brachiaria spp. em ovinos, Prosopis juliflora em bovinos e caprinos, Nerium oleander em bovinos, Opuntia ficus-indica em caprinos e Turbina cordata em equinos e caprinos.

INDEX TERMS: Plantas tóxicas, intoxicações por plantas, bovinos, ovinos, equinos, caprinos, perdas econômicas, semiárido.

\section{INTRODUÇÃO}

As intoxicações por plantas em animais causam perdas econômicas que podem ser definidas como diretas ou indiretas. As perdas diretas são causadas pela morte de animais, diminuição dos índices reprodutivos (aborto, infertilidade, malformações), redução da produtividade nos animais sobreviventes e outras alterações devidas a doenças transitórias, enfermidades sub-clínicas como diminuição da produção de leite, carne ou lã e aumento da suscetibilidade a outras doenças devido à depressão imunológica. As perdas indiretas incluem os custos de controlar as plantas tóxicas nas pastagens, as medidas de manejo para evitar as intoxicações como a utilização de cercas e o pastoreio alternativo, a redução do valor de forragem devido ao atraso na sua utilização, a redução do valor da terra, a compra de gado para substituir os animais mortos, e os gastos associados aos diagnósticos das intoxicações e ao tratamento dos animais afetados (Riet-Correa \& Medeiros 2001, James 1994, Riet-Correa et al. 2007). As perdas diretas por morte de animais podem ser estimadas se há dados de laboratórios de diagnóstico referentes à frequência das diferentes doenças.

No Brasil são conhecidas 113 plantas tóxicas (RietCorrea et al. 2007), das quais 35 são encontradas no Estado da Paraíba. Neste país, com um rebanho de 160.000 .000 de bovinos, as perdas por mortes devidas a intoxicações por plantas são estimadas em 1.120.000 cabeças, o que corresponde a $\mathrm{R} \$ 448.000 .000,00$ considerando o preço médio de $\mathrm{R} \$ 400,00$ por animal (RietCorrea \& Medeiros 2001, Riet-Correa et al. 2007).
Os objetivos deste trabalho foram: relatar as intoxicações por plantas diagnosticadas no Laboratório de Patologia Veterinária (LPV), do Hospital Veterinário (HV) da Universidade Federal de Campina Grande (UFCG); apresentar dados epidemiológicos, clínicos e patológicos das principais intoxicações; e estimar as perdas causadas por mortes de animais intoxicados por plantas na Paraíba.

\section{MATERIAL E MÉTODOS}

Foi feito levantamento dos diagnósticos realizados em ruminantes e equinos no Laboratório de Patologia Veterinária (LPV) do Hospital Veterinário (HV) da UFCG, Campus de Patos, de janeiro de 2000 a dezembro de 2007. Com base em dados dos livros de registro e fichas das necropsias foi realizada a contagem geral dos diagnósticos em cada espécie animal e, posteriormente, dos diagnósticos de intoxicações por plantas. Para a descrição das intoxicações diagnosticadas, os dados epidemiológicos e as lesões de necropsia foram coletados nas fichas do LPV e as lesões histológicas foram estudadas em lâminas histológicas arquivadas ou preparadas posteriormente para esse fim.

Neste trabalho considerou-se como "diagnóstico" a identificação de uma doença ocorrida numa fazenda, seja um caso individual ou coletivo. Casos coletivos (surtos) foram considerados como um só diagnóstico. Considerou-se como "material recebido" um ou mais animais doentes ou mortos, ou uma ou mais amostras (vísceras, sangue, suabes, leite, etc.) procedentes de um mesmo surto.

Para estimar as perdas econômicas que ocorrem na Paraíba em conseqüência das intoxicações por plantas, a freqüência das doenças, nas diferentes espécies, encontradas na área de influência do Hospital Veterinário (que inclui alguns municípios dos Estados de Pernambuco e Rio Grande do Norte) foi considerada como sendo dados da Paraíba. Foram utilizados os dados dos rebanhos e mortalidade dos mesmos, encontrados no site do IBGE (2009) e em artigos publicados anteriormente.

\section{RESULTADOS}

\section{Diagnósticos de intoxicações por plantas, realizados no LPV no período 2000-2007}

De 2000 a 2007 foram realizadas 353 necropsias em bovinos, sendo 284 com diagnóstico e 69 inconclusivos. Dos 284 casos, 21 foram diagnosticados como intoxicação por plantas, representando $7,4 \%$ dos diagnósticos realizados em bovinos. As plantas responsáveis pelos surtos foram Centhraterum brachylepis (um surto), Brachiaria sp. (um surto), Crotalaria retusa (dois surtos), Ipomoea batatas (um surto), Marsdenia sp. (um surto), gramíneas contendo nitratos e nitritos (um surto por Echinochloa polystachya e dois por Pennisetum purpureum), Palicourea aeneofusca (um surto), Prosopis juliflora (três surtos), Nerium oleander (um surto) e Mimosa tenuiflora (sete surtos). Em relação à espécie ovina foram recebidos, de 2000-2007, 292 materiais, sendo 45 inconclusivos e 247 com diagnóstico. Dos casos com diagnóstico, 33 $(13 \%)$ foram surtos de intoxicações por plantas. Os surtos foram causados por Ipomoea asarifolia (quatro surtos), Brachiaria spp. (três surtos), Crotalaria retusa (dois surtos), Tephrosia cinerea (dois surtos), Panicum dicho- 
tomiflorum (um surto), Mascagnia rigida (um surto) e malformações associadas à Mimosa tenuiflora (20 surtos).

No período estudado, 312 casos em caprinos tiveram diagnóstico conclusivo e 83 foram inconclusivos. Dos 312 diagnósticos, 6,4\% corresponderam à intoxicação por plantas. Sete surtos foram causados por Mimosa tenuiflora, um por Ipomoea asarifolia, um por Ipomoea carnea, um por Ipomoea riedelli, três por Prosopis juliflora, um por Arrabidaea corallina, dois por Aspidosperma pyrifolium, e dois por Turbina cordata.

$\mathrm{Na}$ espécie equina foram realizados 97 diagnósticos durante o referido período e 12 materiais ficaram sem diagnóstico. Dos 97 diagnósticos, 13 (14\%) corresponderam a intoxicações por plantas, sendo 12 surtos de intoxicação por Crotalaria retusa e um de intoxicação por Turbina cordata.

Entre ruminantes e equinos foram realizados ao todo 940 diagnósticos no LPV entre 2000-2007. Desses, 85 tiveram o diagnóstico de intoxicação por plantas representando $9 \%$ dos diagnósticos realizados.

\section{Relato de algumas das intoxicações observadas du- rante o período}

Intoxicação por Crotalaria retusa em bovinos. Um surto de intoxicação por Crotalaria retusa foi diagnosticado em fevereiro de 2001 no município de São José do Bonfim. Um bovino adulto, de um total de seis, deu entrada no HV apresentando um quadro clínico inicial de fotossensibilização, sendo que as lesões de pele diminuíam quando o animal permanecia na sombra; no entanto, o quadro evoluiu para cegueira (ceratite) com muito prurido cutâneo e edema de barbela. Na bioquímica hepática 0 animal apresentou uma elevada taxa de GGT. A doença teve uma evolução de três meses até que o animal foi eutanasiado. Na necropsia o fígado estava mais firme. Havia nódulos de até $2 \mathrm{~mm}$ na mucosa da vesícula biliar. $\mathrm{Na}$ histologia observaram-se fibrose periportal acentuada com proliferação de células dos ductos biliares, pericolangite, megalocitose discreta e degeneração de hepatócitos. Grande quantidade da planta sementando foi observada na propriedade. Em 2007, no mês de maio, no município de Soledade, de um total de 20 bovinos, cinco adoeceram e dois morreram. Os animais apresentavam um quadro de fotossensibilização com perda de apetite evoluindo para morte em 2-5 dias. As lesões macroscópicas eram semelhantes às observadas no caso anterior. $\mathrm{Na}$ histologia observaram-se fibrose difusa em ponte, megalocitose e proliferação de células dos ductos biliares. Na propriedade existia uma área severamente invadida pela planta.

Intoxicação por Brachiaria spp. em ovinos. Em 2006, no município de Tabira, Pernambuco, de 300 caprinos e ovinos de uma propriedade, 80 adoeceram e 50 morreram. Os ovinos, na maioria pretos, apresentaram um quadro de irritabilidade, movimentos continuados da cabeça, fotofobia, corrimento ocular purulento e cegueira, evoluindo para morte em até um mês. Segundo o pro- prietário alguns caprinos apresentaram quadro clínico semelhante. Foram necropsiados dois ovinos, de aproximadamente um ano de idade. Na necropsia observaramse tecido subcutâneo ictérico, fígado de coloração alaranjada, vísceras ictéricas, rim escuro e com petéquias na região subescapular, urina amarelada e opacidade de córnea. Na histologia observaram-se, no fígado, necrose individual de hepatócitos e discreta a moderada vacuolização e presença de glóbulos eosinófilicos nos mesmos. Havia múltiplos e dispersos macrófagos espumosos, discreta hiperplasia de células epiteliais de ductos e desorganização dos cordões de hepatócitos. Nos rins havia degeneração e necrose de células epiteliais tubulares com dilatação de túbulos na região cortical externa e presença de cilindros e gotas hialina.

Outro surto de intoxicação por Brachiaria decumbens foi diagnosticado em setembro de 2007, no município de Nova Floresta. O produtor adquiriu 80 animais da espécie ovina, na faixa etária entre um e três meses, e os colocou em uma área com $B$. decumbens. Um mês depois, os animais começaram a adoecer apresentando edema das orelhas e pálpebras, ceratoconjuntivite e cegueira, urina amarela-escura e mucosas ictéricas. Balançavam a cabeça e lambiam o espelho nasal. Todos os 16 animais que adoeceram morreram em 24-72 horas após o início dos sinais clínicos. Após as mortes, o proprietário retirou os animais da pastagem de $B$. decumbens, para uma área onde não tinha o capim e não ocorreram novos casos. As lesões macroscópicas e histológicas foram semelhantes as do surto descrito anteriormente.

Intoxicação por Prosopis juliflora em bovinos e caprinos. Foram diagnosticados três casos de intoxicação por $P$. juliflora em bovinos, um no ano de 2000 acometendo uma vaca de oito anos de idade, outro em 2001 em um bovino de três anos, e outro em 2007 em um bovino de dois anos e meio. Todos os animais tinham tido acesso a grande quantidade de vagens de algaroba no campo ou eram alimentados quase que exclusivamente com vagens da planta e apresentavam sinais característicos da doença. Macroscopicamente, o músculo masseter apresentava-se diminuído de volume e de coloração amarelada ou esbranquiçada. $\mathrm{Na}$ histologia, os músculos masseter, bucinador, temporal e pterigoideo medial apresentavam atrofia neurogênica e substituição de fibras musculares por tecido fibroso ou gordura. Em raros neurônios dos núcleos motores do trigêmeo e outros núcleos do tronco encefálico havia vacuolização fina do pericário e desaparecimento da substância de Nissl. Em todos os casos, além da atrofia neurogênica, o achado histológico mais consistente foi degeneração walleriana nas raízes intracranianas dos nervos cranianos, principalmente do trigêmeo. Em um dos bovinos não se observaram neurônios vacuolizados, mas havia uma aparente perda de neurônios e astrocitose, evidenciada pela detecção por imunohistoquímica da proteína fibrilar ácida (GFAP). Não está esclarecido se a dificuldade em observar neurônios vacuolizados na intoxicação por vagens de $P$. juliflora em 
bovinos deve-se à regressão dessas lesões, à perda de neurônios ou à dificuldade de observar os mesmos em cortes de rotina, pois aparentemente são mais bem vistos em cortes ultrafinos.

Três surtos foram diagnosticados em caprinos, um deles foi descrito por Lima et al. (2004). Um surto afetou um caprino em 2006 no município de Taperoá. Outro surto, que ocorreu na mesma fazenda de Taperoá, afetou dois caprinos adultos. Nesta fazenda ocorrem casos esporádicos da intoxicação em caprinos em um rebanho de aproximadamente 1200 animais. Os animais tinham acesso à planta e ingeriam as vagens no pasto. As intoxicações aconteciam em qualquer época do ano. A doença afetava exclusivamente caprinos adultos e, dentre esses, os mais velhos. Os sinais clínicos foram dificuldade de apreender e manter alimentos na boca, diminuição do tônus da mandíbula e língua, ruminação prolongada, freqüente e com um barulho característico ocasionado pela batida dos dentes, salivação profusa, diminuição do volume dos músculos masseteres e emagrecimento. Na necropsia os músculos masseter e temporal apresentavam-se diminuídos de volume e amarelados caracterizando atrofia muscular. $\mathrm{Na}$ histologia observava-se vacuolização fina e discreta de alguns neurônios do núcleo motor do trigêmeo e, rara- mente, em outros núcleos do tronco encefálico com presença de alguns esferóides axonais. Havia degeneração walleriana das raízes intracranianas dos nervos trigêmeo e oculomotor. Nos músculos afetados foi observada atrofia neurogênica de fibras musculares, fibrose e infiltrado inflamatório.

Intoxicação por Nerium oleander em bovinos. Foi diagnosticada, em dezembro de 2007 intoxicação em bovinos por N. oleander (Fig.1A). Quatro bovinos adoeceram, dos quais dois morreram. Os animais se alimentaram pela manhã com uma planta que, segundo os proprietários, é conhecida popularmente como "mata fome" e junto com ela receberam também galhos e folhas de $N$. oleander. No período da tarde do mesmo dia os bovinos apresentaram andar cambaleante, polidipsia e desidratação. Após 72 horas do início dos sinais um bovino de um ano de idade sem raça definida morreu e foi necropsiado. O animal apresentava bom estado corporal e petéquias no tecido subcutâneo, nas regiões submandibular, cervical e abdominal, nos músculos intercostais, no omento, na serosa do rúmen, na pleura parietal e no epicárdio (Fig.1B). Na mucosa da traquéia havia hemorragias focais e o pulmão apresentava-se congesto e com edema. No abomaso observavam-se áreas focais na superfície da mucosa

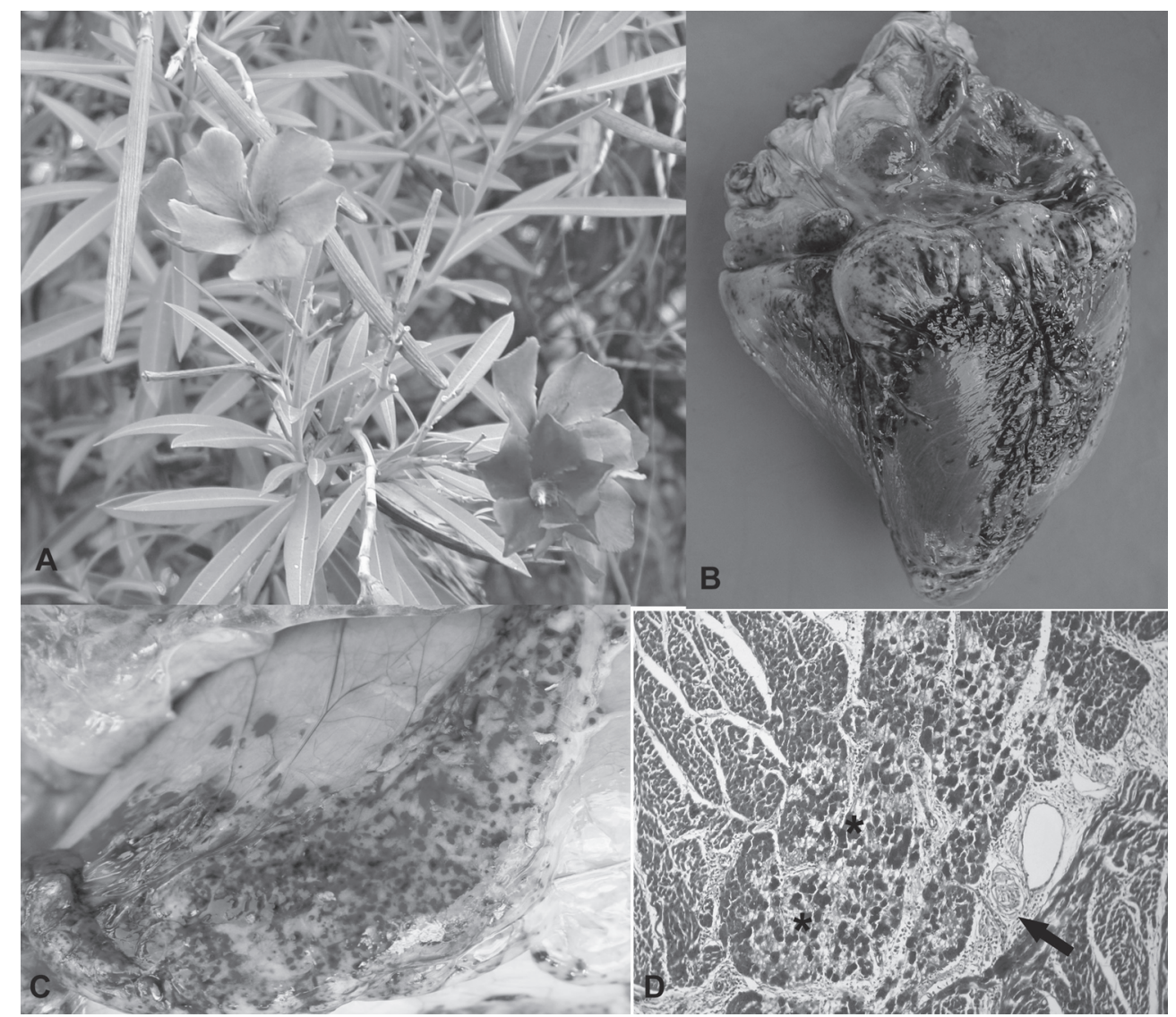

Fig.1. (A) Nerium oleander. Município de Patos, Paraíba. (B-D) Bovino intoxicado por $N$. oleander mostrando (B) severas hemorragias no epicárdio, (C) no saco pericárdico; (D) focos de necrose de fibras cardíacas (asteriscos) associados a infiltrado mononuclear (seta) nos músculos papilares do miocárdio. HE, obj.20x. 
com coloração avermelhada (sufusões), que se estendiam para a porção inicial do intestino delgado. A superfície renal apresentava-se amarelo-pálido e ao corte observavam-se petéquias na região medular. No coração observaram-se múltiplas hemorragias, petéquias e sufusões no saco pericárdico (Fig.1C) e no endocárdio. Microscopicamente, o coração apresentava, nos músculos papilares dos ventrículos direito e esquerdo, áreas extensas e multifocais de degeneração e necrose das fibras musculares com infiltrado inflamatório mononuclear associado a hemorragias (Fig.1D). No fígado havia áreas focais de degeneração e necrose de hepatócitos, com hemorragia, congestão e morte individual de hepatócitos.

Intoxicação associada à ingestão de Opuntia ficusindica em caprinos. Mortalidade de caprinos associada à ingestão de Opuntia ficus-indica (palma) foi observada num rebanho de 95 caprinos e 15 ovinos, no município de São José dos Cordeiros, Paraíba. O proprietário relatou que de janeiro a março de 2008 morreram 33 caprinos e havia dois doentes, a maioria com idade entre dois e três anos e quatro menores de um ano, sem distinção de sexo. Durante a visita à propriedade observou-se que os animais estavam pastejando em um cultivo de palma e que toda a rebrota da planta tinha sido consumida ou estava sendo consumida pelos animais. Exceto a palma o resto da vegetação estava seca e com muito pouca disponibilidade de forragem. Um caprino de oito meses de idade apresentou apatia, anorexia, diarréia, sensibilidade à palpação abdominal, desidratação moderada, mucosas congestas e incoordenação dos membros posteriores. Exames parasitológicos de fezes tiveram resultado negativo, a urina tinha densidade baixa $(1,006)$, o hemograma apresentou leucocitose $(22.150 / \mu \mathrm{l})$ acompanhada de neutrofilia ( $75 \%$ ) e linfopenia (23\%). A uréia sérica estava aumentada (53mg/dl) e culturas bacteriológicas de matérias fecais foram negativas para Salmonella spp. Após cinco dias alimentando-se com capim elefante (Pennisetum purpureum) e água a vontade o animal apresentou melhora clínica. Os níveis de oxalatos totais na planta foram de $5,63 \%{ }^{3}$.

Intoxicação por Turbina cordata. Em dezembro de 2006, no município de Juazeiro, Bahia ocorreram dois surtos de intoxicação por Turbina cordata em caprinos e um em equinos. Os dois equinos da fazenda apresentavam sinais nervosos incluindo tremores, ataxia severa, estação com os membros abertos, alteração de comportamento e emagrecimento há aproximadamente 32 meses (Fig.2). Quando puxado pelo cabresto apresentava relutância em caminhar, com saltos e quedas.

Existia grande quantidade da planta na fazenda. O proprietário relatou que já tinham ocorrido casos em caprinos, os quais apresentavam ataxia, tremores de lábios, que se intensificavam após a agitação, hipermetria, relutância em caminhar, emagrecimento e morte. O diagnós-

\footnotetext{
${ }^{3}$ Determinação realizada pela Profa. Valéria Duarte Cerqueira, Universidade Federal do Pará.
}

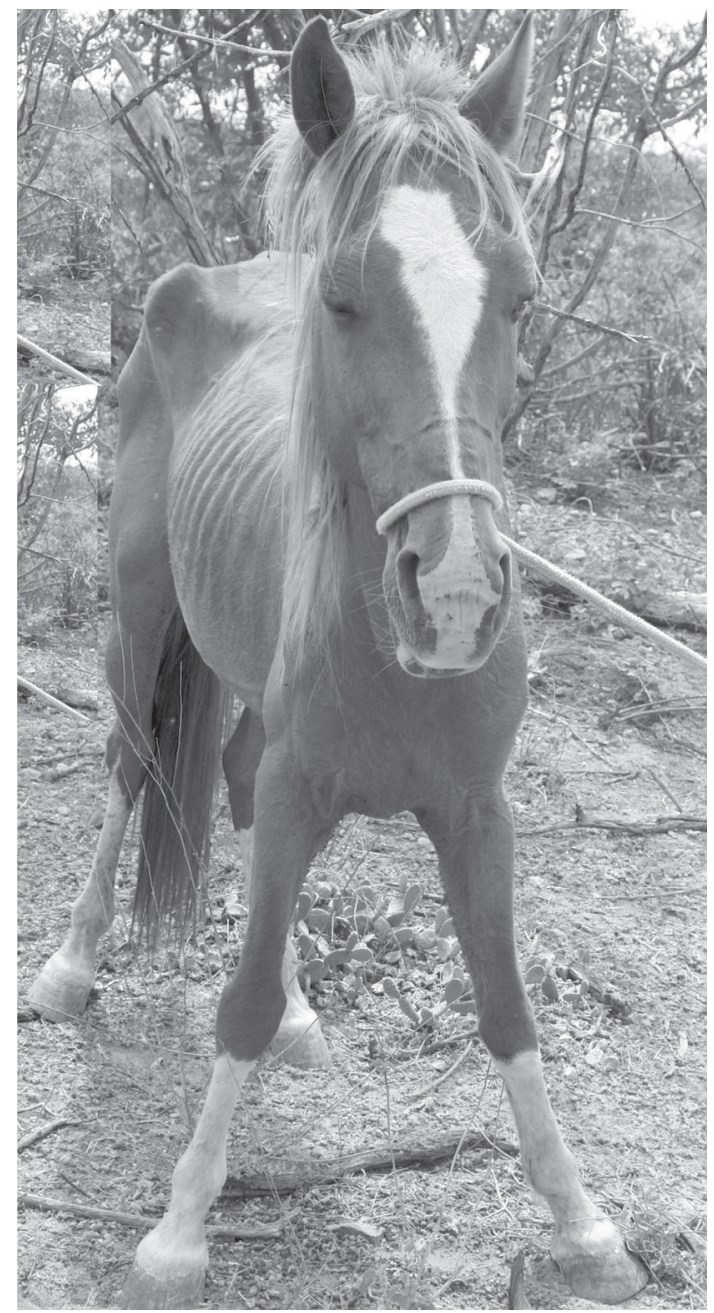

Fig.2. Equino intoxicado por Turbina cordata em estação com os membros abertos e acentuado emagrecimento.

tico foi realizado através dos dados clínicos e epidemiológicos. Na mesma época outros dois surtos foram diagnosticados em caprinos que apresentavam sinais clínicos e lesões histológicas características (Dantas et al. 2007).

Outras intoxicações diagnosticadas durante o período foram publicadas separadamente e são mencionadas a seguir: intoxicação por Ipomoea batatas mofada em bovinos (Medeiros et al. 2001), intoxicação por Crotalaria retusa em ovinos (Dantas et al. 2004, Nobre et al. 2004b, 2005), intoxicação por Crotalaria retusa em equinos (Nobre et al. 2004a,b), intoxicação por Echinochloa polystachya e por Pennisetum purpureum em bovinos (Medeiros et al. 2003b), intoxicação por Panicum dycothomiflorum em ovinos (Riet-Correa et al. 2009), intoxicação por Tephrosia cinerea em ovinos (Santos et al. 2007), intoxicação por Marsdenia sp. em bovinos (RietCorrea et al. 2004), intoxicação por Palicourea aeneofusca em bovinos (Vasconcelos et al. 2008a), intoxicação por Mascagnia rigida em ovinos (Vasconcelos et al. 2008b), intoxicação por Arrabidaea corallina em caprinos 
(Pessoa et al. 2006), intoxicação por Ipomoea asarifolia e Ipomoea carnea em ovinos e caprinos (Medeiros et al. 2003a, Guedes et al. 2006) e intoxicação por Ipomoea riedelli em caprinos (Barbosa et al. 2006).

\section{DISCUSSÃO E CONCLUSÕES}

No Brasil, a estimativa de mortalidade anual da espécie bovina é de aproximadamente 5\% (Corrêa et al. 2000) e na Paraíba a população atual de bovinos é de 1.052 .613 cabeças. Considerando o percentual de 5\% acima mencionado, a perda de bovinos na Paraíba, representa cerca de 52.630 animais. Considerando que $7,4 \%$ dessas mortes são causadas por plantas tóxicas, estima-se que 3.895 bovinos morrem por intoxicações por plantas. Considerando um preço médio de $\mathrm{R} \$ 400,00$ por animal, estima-se uma perda econômica de $\mathrm{R} \$ 1.557 .848,00$.

O rebanho de ovinos na Paraíba é de 411.669 cabeças. A taxa de mortalidade anual nesta espécie, na Paraíba, é de $15,18 \%$ (Lobo 2002), isso significa uma mortalidade anual de 62.491 ovinos. Usando-se o percentual de mortes por plantas obtido neste estudo (13\%) para esta espécie pode-se considerar que anualmente morrem por intoxicação por plantas 8.374 ovinos. Multiplicando esse valor por um valor estimativo de $\mathrm{R} \$ 60,00$ por animal, a perda econômica por mortes, nesta espécie, pode ser estimada em $\mathrm{R} \$ 502.428,00$.

Para caprinos a taxa de mortalidade anual na Paraíba não foi encontrada. Considerando-se uma mortalidade semelhante à dos ovinos $(15,18 \%)$ e como a população de caprinos na Paraíba é de 657.824, estima-se que 99.857 caprinos morrem anualmente. Considerando que $6,4 \%$ destas mortes são causadas por plantas tóxicas, pode-se estimar que 6.390 caprinos morrem anualmente por esta causa. Considerando-se um valor de $R \$ 50,00$ por animal a perda anual por mortes pode ser estimada em $R \$ 319.500$.

A população de equinos na Paraíba é de 50.910 cabeças, mas não há dados referentes à mortalidade anual nesta espécie. No entanto, levando em consideração uma mortalidade de $5 \%$, calcula-se que anualmente morrem 2.545 equinos. Considerando que $14 \%$ dos equinos morrem intoxicados por plantas, estima-se que 366 equinos morrem por ano por essa causa. Utilizando-se como base o valor de $R \$ 400,00$ por cabeça, a perda econômica para esta espécie seria em torno de $\mathrm{R} \$ 146.562,00$.

Com base nos dados obtidos, estima-se que as intoxicações por plantas no estado da Paraíba em ruminantes e equinos representam uma perda econômica anual, por morte de animais, de $R \$ 2.733 .097,00$, o que justifica plenamente investir na divulgação de medidas preventivas, além da realização de pesquisas para melhor conhecimento das intoxicações e determinação das formas mais adequadas de controle e profilaxia. No entanto, em relação à mortalidade de bovinos por plantas tóxicas, as cifras encontradas ( $9 \%$ do total de mortes por plantas em relação às outras doenças) foram menores que a de outros estados. No Rio Grande do Sul, Santa Catarina e
Bahia essas mortes são estimadas em 10\% a 14\% (RietCorrea \& Medeiros 2001, Riet-Correa et al. 2007, Rissi et al. 2007).

Embora este levantamento tenha registrado somente um surto de intoxicação por Mascagnia rigida em ovinos, a intoxicação é importante em bovinos na região, como demonstrado por trabalhos de levantamento junto a produtores (Silva et al. 2006, Assis et al. 2009). O reduzido número de surtos dessa intoxicação observado neste estudo, provavelmente, deve-se ao fato de que a intoxicação é bem conhecida pelos produtores, que não consultam veterinários quando ocorrem surtos; ou por que os veterinários de campo não enviam material ao laboratório para a confirmação do diagnóstico. Essa confirmação é relevante para a determinação da importância das intoxicações por plantas, pois intoxicações importantes, como as que causam mortes súbitas, podem ser sub-diagnosticadas, enquanto que as perdas causadas por outras plantas menos importantes podem ser superestimadas. Portanto, os levantamentos já realizados na Paraíba (Silva et al. 2006, Assis et al. 2009), tanto quanto os dados dos laboratórios de diagnóstico devem ser considerados para o conhecimento das intoxicações e estimar as perdas ocasionadas.

Mimosa tenuiflora, que causa malformações em ruminantes, é responsável por importantes perdas econômicas no rebanho da Paraíba. Estudos recentes estimam que na região Nordeste morrem anualmente 273.120 cabritos e 259.582 cordeiros por malformações (Riet-Correa et al. 2007). A reprodução experimental das malformações e a descrição das mesmas foram publicadas anteriormente (Pimentel et al. 2007, Nóbrega et al. 2004, Medeiros et al. 2005).

Outras intoxicações importantes na região são causadas por Ipomoea asarifolia em ruminantes (Medeiros et al. 2003a, Guedes et al. 2006, Araújo et al. 2008) e a intoxicação por Crotalaria retusa em equídeos (Nobre et al. 2004a). Apesar de amplamente conhecida pelos produtores, a intoxicação por Prosopis juliflora ainda causa, aparentemente, perdas econômicas importantes no semiárido; no entanto, por constituir uma importante fonte de alimento para os animais e ser uma planta de difícil controle, medidas preventivas devem ser tomadas para que possa ser utilizada na alimentação animal sem riscos de intoxicação (Riet-Correa et al. 2006).

O diagnóstico de intoxicação por Nerium oleander alerta para a possível importância dessa intoxicação no Nordeste onde é frequente durante a seca a alimentação dos animais com restos de árvores e plantas de jardins após a poda. Diversos surtos desta intoxicação foram descritos em bovinos no Rio Grande do Norte (Silva et al. 2006, Soto Blanco et al. 2006). Outro surto foi registrado na região do Vale do Piancó, Paraíba, onde 20 animais adoeceram e quatro morreram após a ingestão da planta que tinha sido cortada junto a outras árvores e fornecida aos animais (Rodrigues 2008).

Um surto de intoxicação ocorreu em caprinos pastejando em uma plantação de Opuntia fícus-indica (palma). 
Pela sua resistência à seca e facilidade de ser produzida com pouca disponibilidade de água e por ser uma ótima fonte de energia esta é uma forrageira de extrema importância para a pecuária do nordeste. Apesar de não ter sido comprovado mediante necropsia que a intoxicação fosse causada por essa planta, o alto conteúdo de oxalatos $(5,63 \%)$ da mesma sugere o diagnóstico de intoxicação por oxalatos. Concentrações de oxalato de 4-8\% são consideradas tóxicas para bovinos (Jones \& Ford 1972) e $O$. ficus-indica tem sido responsabilizada por intoxicações por oxalatos (Kellerman et al. 2005). Outro fator que sugere a intoxicação é que os caprinos tinham sido soltos na plantação e tiveram acesso à vontade aos brotos da planta que são os mais ricos em oxalatos.

Os resultados deste trabalho complementam a informação gerada anteriormente sobre plantas tóxicas da Paraíba e, se analisados junto a outros levantamentos sobre plantas tóxicas da região (Silva et al. 2006, Assis et al. 2009), possibilitam um bom conhecimento sobre epidemiologia, diagnóstico e formas de controle das intoxicações.

Agradecimentos.- Ao CNPq pela concessão da bolsa do mestrado. Este trabalho foi financiado pelo Programa de Apoio a Núcleos de Excelência (Pronex, Proc.001/04, CNPq, FAPESQ, MCT), pelo programa Institutos do Milênio (Proc.420012/2005-2, CNPq), e pelo INCT para o controle das intoxicações por plantas (Proc.573534/2008-0, CNPq).

\section{REFERÊNCIAS}

Assis T.S., Medeiros R.M.T., Riet-Correa F., Araújo J.A.S. \& Dantas A.F.M. 2009. Intoxicações por plantas em ruminantes e equídeos no sertão paraibano. Pesq. Vet. Bras. 29(11):919-924.

Araújo J.A.S., Riet-Correa F., Medeiros R.M.T., Soares M.P., Oliveira D.M. \& Carvalho F.K.L. 2008. Intoxicação experimental por Ipomoea asarifolia em caprinos e ovinos. Pesq. Vet. Bras. 28(10):488-494.

Barbosa R.C., Riet-Correa F., Medeiros R.M.T., Lima E.F., Barros S.S., Gimeno J.E., Molyneux R.J. \& Gardner D.R. 2006. Intoxication by Ipomoea sericophylla and Ipomoea riedelii in goats in the state of Paraíba, northeastern Brazil. Toxicon 47:371-379.

Corrêa S.E., Vieira A., Costa P.F. \& Cezar M.I. 2000. Sistema semiintensivo de produção de carne de bovinos Nelore no Centro-Oeste do Brasil. Embrapa Gado de Corte, Campo Grande, MS, p.5.

Dantas A.F.M., Nobre V.M.T., Riet-Correa F., Tabosa I.M., Júnior G.S., Medeiros J.M., Silva R.M.N., Silva E.M.N., Anjos B.L. \& Medeiros J.K.D. 2004. Intoxicação crônica espontânea por Crotalaria retusa (Fabaceae) em ovinos na região do semiárido Paraibano, Brasil. Pesq. Vet. Bras. 24(Supl.):18-19.

Dantas A.F.M., Riet-Correa F., Gardner D.R., Medeiros R.M.T., Barros S.S., Anjos B.L. \& Lucena R.B. 2007. Swainsonine-induced lysosomal storage disease in goats caused by the ingestion of Turbina cordata in northeastern Brazil. Toxicon 49:11-16.

Guedes K.M.R., Riet-Correa F., Dantas A.F.M., Simões S.V.D., Miranda Neto E.G., Nobre V.M.T. \& Medeiros R.M.T. 2006. Doenças do sistema nervoso central em caprinos e ovinos no semiárido. Pesq. Vet. Bras. 27(1):25-34.

IBGE 2009. Pesquisa Pecuária Municipal. Instituto Brasileiro de Geografia e Estatística, Rio de Janeiro. Disponível em: http://IBGE.gov.br Acesso em 10 de janeiro de 2009.

James L.F. 1994. Solving poisonous plant problems by a team approach, p.1-6. In: Colegate S.M. \& Dorling P.R. (Eds), Plant Associated Toxins. $\mathrm{CAB}$ International, Wallingford.
Jones R.J. \& Ford C.W. 1972. The soluble oxalate content of some tropical pasture grasses grown in south-east Queensland. Tropical Grasslands 6(3):201-204.

Kellerman T.S., Coetzer J.A.W., Naudé T.W. \& Botha C.J. 2005. Plant Poisonings and Mycotoxycoses. Oxford University Press, Cape Town, p.221-224.

Lima E., Riet-Correa F., Amorin S.L. \& Sucupira Júnior G. 2004. Intoxicação por favas de Prosopis juliflora (algaroba) em caprinos no Nordeste do Brasil. Pesq. Vet. Bras. 24(Supl.):36-37.

Lobo R.N.B. 2002. Melhoramento genético de caprinos e ovinos: desafios para o mercado. Anais VI Seminário Nordestino de Pecuária (PECNORDESTE), Fortaleza, CE, p.44-60.

Medeiros R.M.T., Simões S.V.D., Tabosa I.M., Nóbrega W.D. \& RietCorrea F. 2001. Bovine atypical instertitial pneumonia associated with the ingestion of damaged sweet potatoes (Ipomoea batatas) in northeastern Brazil. Vet. Hum. Toxicol. 43(4):205-207.

Medeiros R.M.T., Barbosa R.C., Riet-Correa F., Lima E.F., Tabosa I.M., Barros S.S., Gardner D.R. \& Molyneux R.J. 2003a. Tremorgenic syndrome in goats caused by Ipomoea asarifolia in northeastern Brazil. Toxicon 41:933-935.

Medeiros R.M.T., Riet-Correa F., Tabosa I.M., Silva Z.A., Barbosa R.C. Marques A.V.M.S. \& Nogueira F.R.B. 2003b. Intoxicação por nitratos e nitritos em bovinos por ingestão de Echinochloa polystachya (capim-mandante) e Pennisetum purpureum (capim-elefante) no sertão da Paraíba. Pesq. Vet. Bras. 23(1):17-20.

Medeiros J.M., Tabosa I.M., Simões S.V.D., Nóbrega Junior J.E., Vasconcelos J.S. \& Riet-Correa F. 2005. Mortalidade perinatal em cabritos no semiárido da Paraíba. Pesq. Vet. Bras. 25(4):201-206.

Nobre V.M.T., Riet-Correa F., Barbosa Filho J.M., Tabosa I.M. \& Vasconcelos J.S. 2004a. Intoxicação por Crotalaria retusa (Fabaceae) em eqüídeos no semiárido da Paraíba. Pesq. Vet. Bras. 24:132-143.

Nobre V.M.T., Riet-Correa F., Dantas A.F.M., Tabosa I.M., Medeiros R.M.T. \& Barbosa Filho J.M. 2004b. Intoxication by Crotalaria retusa in ruminants and equidae in the state of Paraíba, northeaster Brazil, p.275-279. In: Acamovich T., Stewart C.S. \& Pennycott T.W. (Eds), Plant Poisoning and Related Toxins. CAB International, Glasgow, UK.

Nobre V.M.T., Dantas A.F.M., Riet-Correa F., Barbosa Filho J.M., Tabosa I.M. \& Vasconcelos J.S.V. 2005. Acute intoxication by Crotalaria retusa in sheep. Toxicon 45:347-352.

Nóbrega R., Riet-Correa F., Nóbrega R.S., Correia A.P., Aragão M.C., Medeiros R.M. \& Tabosa I.M. 2004. Malformações ósseas em ovinos na região semiárida do nordeste do Brasil. Pesq. Vet. Bras. 24(Supl.):45.

Pessoa A.F.A., Pessoa C.R.M., Araújo J.A.S., Medeiros R.M.T., Dantas A.F. \& Riet-Correa F. 2006. Diarréia em caprinos do Cariri Paraibano associada à ingestão de Arrabidaea sp. XIII Encontro Nacional de Patologia Veterinária. $C D$.

Pimentel L.A., Riet-Correa F., Gardner D.R., Panter K., Dantas A.F.M., Medeiros R.M.T., Mota R.A. \& Araújo J.A.S. 2007. Mimosa tenuiflora as a cause of malformations in ruminants in the northeastern Brazilian semiarid rangelands. Vet. Pathol. 44:928-931.

Riet-Correa F. \& Medeiros R.M.T. 2001. Intoxicação por plantas em ruminantes no Brasil e no Uruguai: importância econômica, controle e riscos para a saúde pública. Pesq. Vet. Bras. 21:38-42.

Riet-Correa F., Silva D.M., Oliveira O.F., Simões S.V.D., Medeiros R.M.T. \& Nóbrega R.S. 2004. Intoxicação por Marsdenia spp. (mata-calado) em ruminantes. Pesq. Vet. Bras. 24 (Supl.):50-51.

Riet-Correa F., Medeiros R.M.T. \& Dantas A.F.M. 2006. Plantas Tóxicas da Paraíba. Centro de Saúde e Tecnologia Rural, Patos. SEBRAE/ PB, p.9-58.

Riet-Correa F., Medeiros R.M.T., Tokarnia C.H. \& Döbereiner J. 2007. Toxic plants for livestock in Brazil: Economic impact, toxic species, control measures and public health implications, p.2-14. In: Panter 
K.E., Wierenga T.L. \& Pfister J.A. (Eds), Poisonous Plants: Global research and solutions. CAB International, Wallingford.

Riet-Correa F., Haraguchi M., Dantas A.F.M., Burakovas R.G., Medeiros R.M.T. \& Matos P.F. 2009. Sheep poisoning by Panicum dichotomiflorum in northeastern Brazil. Pesq. Vet. Bras 29(1):94-98.

Rissi D.R., Rech R.R., Pierezan F., Gabriel A.L., Trost M.E., Brum J.S., Kommers G.C. \& Barros C.S.L. 2007. Intoxicações por plantas e micotoxinas associadas a plantas em bovinos no Rio Grande do Sul: 461 casos. Pesq. Vet. Bras. 27:261-268.

Rodrigues A. 2008. Comunicação pessoal (Médica Veterinária, Piancó, PB).

Santos C.A.J., Riet-Correa F., Dantas A.F.M., Barros S.S., Molyneux R.J., Medeiros R.M.T., Silva D.M. \& Oliveira O.F. 2007. Toxic hepatopathy in sheep associated with the ingestion of the legume Tephrosia cinerea. J. Vet. Diag. Invest. 19:690-694.
Silva D.M., Riet-Correa F., Medeiros R.M.T \& Oliveira O.D. 2006. Plantas tóxicas para ruminantes e eqüídeos no Seridó Ocidental e Oriental do Rio Grande do Norte. Pesq. Vet. Bras. 26(4):223-236.

Soto Blanco B., Fontenele-Neto J.D., Silva D.M., Reis P.F.C.C. \& Nóbrega J.E. 2006. Acute cattle intoxication from Nerium oleander pods. Trop. Anim. Health Prod. 38:451-454.

Vasconcelos J.S., Riet-Correa F., Dantas A.F.M., Medeiros R.M.T \& Dantas A.J. 2008a. Mortes súbitas em bovinos causadas por Palicourea aeneofusca (Rubiaceae) e Mascagnia rigida (Malpighiaceae) na Zona da Mata Paraibana. Pesq. Vet. Bras. 28:457460.

Vasconcelos J.S., Riet-Correa F., Dantas A.F.M., Medeiros R.M.T., Galiza G.J., Oliveira D.M. \& Pessoa A.F.A. 2008b. Intoxicação por Mascagnia rigida (Malpighiaceae) em ovinos e caprinos. Pesq. Vet. Bras. 28:521-526. 